\title{
Bayesian binning for maximising information rate of rapid serial presentation for sensory neurons Dominik Endres* and Peter Földiák
}

\author{
Address: School of Psychology, University of St. Andrews, Fife, KY16 9JP, UK \\ Email: Dominik Endres* - dme2@st-andrews.ac.uk \\ * Corresponding author
}

from Sixteenth Annual Computational Neuroscience Meeting: CNS*2007

Toronto, Canada. 7-12 July 2007

Published: 6 July 2007

BMC Neuroscience 2007, 8(Suppl 2):PI5I doi:I0.I I86/I47I-2202-8-S2-PI5 I

(c) 2007 Endres and Földiák; licensee BioMed Central Ltd.

Understanding the response properties of single neurons is seriously limited by the available experimental time and the rate [bit/s] at which information can be gained from the neurons. A substantial improvement in the latter can be achieved by speeding up the presentation of stimuli.

We show how the novel technique of Bayesian Binning [1] can be used to find the optimal stimulus presentation rate of a continuous sequence of stimuli.

This method applied to neurons in high-level visual cortical area STSa gives optimal presentation rates of approximately $56 \mathrm{~ms} / \mathrm{stimulus}$ (18 stimuli/s) which is significantly faster than conventional presentation rates, allowing a better sampling of stimulus space. We relate these results to findings obtained with the Bayesian Bin Classification method $[2,3]$, which can be used to select the optimal time window for the analysis of the continuous response stream. Both methods will soon be freely available as standalone command-line applications or Matlab/Octave plugins.

The optimal window duration is equal to the stimulus duration near the best presentation rate. Interestingly, this duration also corresponds to the peak of spike efficiency [bit/spike] of a rate code whose firing rates match those found in visual neurons (area STSa).

\section{References}

I. Endres D, Földiák P: Bayesian bin distribution inference and mutual information. IEEE Trans Inform Theory 2005, 5 I (II):3766-3779.

2. Endres D: Bayesian and information-theoretic tools for neuroscience. $\mathrm{PhD}$ thesis 2007 [http://hdl.handle.net//0023//62]. University of St. Andrews, digital research repository

3. Endres D, Földiák P: Exact Bayesian bin classification: a fast alternative to Bayesian classification and its application to neural response analysis. 2007 in press. 